\title{
No differences between conservative and surgical management of acromioclavicular joint osteoarthritis: a scoping review
}

\author{
Francesc Soler ${ }^{1}\left[\right.$ C Fabrizio Mocini ${ }^{2} \cdot$ Donald Tedah Djemeto $^{3} \cdot$ Stefano Cattaneo $^{4} \cdot$ Maristella F. Saccomanno $^{2}$. \\ Giuseppe Milano ${ }^{3,4}$
}

Received: 19 September 2020 / Accepted: 13 November 2020

(c) European Society of Sports Traumatology, Knee Surgery, Arthroscopy (ESSKA) 2021

\begin{abstract}
Purpose To conduct a scoping review to clarify the management of acromioclavicular joint osteoarthritis, as well as to identify any existing gaps in the current knowledge.

Methods Studies were identified by electronic databases (Ovid, Pubmed) from their inception up to April 2nd, 2020. All studies reporting functional outcomes after conservative or surgical treatment of acromioclavicular joint osteoarthritis, either primary or secondary to trauma or distal clavicle osteolysis, were included. Following data were extracted: authors, year of publication, study design (prospective or retrospective), LOE, number of shoulders treated conservatively or surgically, patients' age, OA classification, type of conservative treatment, surgical approach, surgical technique, functional outcomes, complications, revisions, and length of follow-up. Descriptive statistics was used. Quality appraisal was assessed through the Cochrane risk of bias tool for LOE I/II studies, while the MINORS checklist was used for LOE III/IV studies.

Results Nineteen studies were included for a total of 861 shoulders. Mean age of participants was $48.5 \pm 7.4$ years. Mean follow-up was $43.8 \pm 29.9$ months. Four studies reported functional results after conservative treatment, whereas 15 studies were focused on surgical management. No studies directly compared conservative and surgical treatment. Seven studies reported a surgical approach after failure of previous conservative treatment. All studies reported functional improvement and pain relief. Complication rate was low. Overall methodological quality of included studies was very low.

Conclusion Conservative and surgical treatments are both effective in acromioclavicular joint osteoarthritis management. However, available data did not allow to establish the superiority of one technique over another.

Level of evidence Level IV.
\end{abstract}

Keywords Acromioclavicular · Osteoarthritis · Injection · Physical therapy · Distal clavicle excision

\section{Introduction}

Electronic supplementary material The online version of this article (https://doi.org/10.1007/s00167-020-06377-8) contains supplementary material, which is available to authorized users.

Francesc Soler

fsoler@traumadvance.es

Fabrizio Mocini

fabriziomocini90@gmail.com

Donald Tedah Djemeto

donalte2@yahoo.fr

Stefano Cattaneo

stefano1cattaneo@gmail.com

Maristella F. Saccomanno

maristellasaccomanno@hotmail.it
Osteoarthritis (OA) is one of the most common cause of disorder of the acromioclavicular (AC) joint in adult patients [11], sometimes resulting in pain and physical limitations

\section{Giuseppe Milano}

giuseppe.milano@unibs.it

1 Traumadvance Orthopaedic Group, Terrassa, Spain

2 Orthopaedics Institute, Fondazione Policilinico A. Gemelli IRCCS, Rome, Italy

3 University of Brescia, Brescia, Italy

4 Department of Bone and Joint Surgery, Spedali Civili, Brescia, Italy 
with overhead and cross-body movements. As a matter of facts, it develops as a consequence of constant stress on the joint, often in people who perform repeated overhead lifting activities [6]. Diagnosis is mainly clinical, corroborated by typical radiographs and MRI findings, such as inferior osteophytes and joint space narrowing [1]. It is also often associated with distal clavicular osteolysis [3]. Although frequently overlooked [35], the prevalence of AC joint OA on MRI has been reported to be as high as $68 \%$ in patients aged 30 years or less, and up to $93 \%$ in patients older than 30 years $[11,44]$. However, most of the time it is asymptomatic or causes mild localised discomfort, becoming severe in only $5 \%$ of cases, without significant difference between the two sexes [44].

AC joint OA often coexists with other shoulder pathologies such as subacromial impingement and rotator cuff tears [46], thus making its treatment algorithm even more debatable.

While the initial treatment is supposed to be conservative especially when $\mathrm{AC}$ joint pain seems to be isolated, a room for a surgical option is still open either way as a first line option or after failure of conservative treatment, regardless the presence of concurrent shoulder pathologies. The mainstay of conservative treatment is composed of: intraarticular injections, physical therapy, activity modifications and non-anti-inflammatory drugs (NSAIDs). From the surgical standpoint, the distal clavicle excision (DCE) represent the final solution [28].

Looking deeper into the current literature on this topic $[8$, 20], the best treatment option to manage isolated AC joint OA has not been clarified yet.

The purpose of the present study was to conduct a scoping review to systematically map the research done in this area, as well as to identify any existing gaps in the current knowledge.

\section{Materials and methods}

A scoping review was carried out according to the PRISMA (Preferred Reporting Items for Systematic reviews and MetaAnalyses) guidelines adapted for scoping reviews [45].

\section{Literature search}

Studies were identified by scanning the main electronic databases (Ovid, PubMed) from their inception to April 2nd, 2020. The search was first applied to MEDLINE through Ovid, and then adapted for PubMed. Search terms were chosen to be unspecific enough to encompass all possibilities for applicable studies. Full search strategies are available in Appendix 1.
All studies reporting functional outcomes after conservative or surgical treatment of AC joint OA, either primary or secondary to trauma or distal clavicle osteolysis, were included in the review (level of evidence, LOE I-IV). The diagnosis of AC joint OA was based on both clinical and radiological findings. On physical examination, patients affected by tenderness to palpation over the $\mathrm{AC}$ joint, pain in the $\mathrm{AC}$ joint with adduction of the arm across the chest, and/or localised AC discomfort with terminal abduction of the shoulder were considered eligible. Studies were included when also reporting imaging findings suggestive of OA, such as: joint space narrowing, sclerosis of the lateral aspect of the acromion and hypertrophic spurs on the superior and inferior aspects of both the acromial and clavicular sides of the joint $[14,40]$. Conservative approach included any combination of non-surgical treatment: physical therapy (rest, activity modification, exercises, massages and manipulation, cryotherapy, heat), NSAIDs, single or multiple local injections of anaesthetic and/or corticosteroids. Surgical treatment included both open and arthroscopic approach.

Only published data on peer review journals were considered. No language restriction was first applied. Titles of journals, names of authors or supporting institutions were not masked at any stage.

Exclusion criteria were: clinical diagnosis of AC joint pain with no imaging, ACJ dislocations, any surgery in which concomitant procedures (rotator cuff repair, capsular shift, and labral repair) were performed, except for subacromial decompression, as it always reported when an arthroscopic indirect approach was chosen. Studies including revision surgery were excluded. Animal studies, biomechanical studies, case reports, technical notes, reviews, expert opinions and editorial pieces were also excluded.

As the functional assessment was the primary outcome, complications and revision rates were also recorded. Any functional outcome measurement reported by each study was included.

Two independent reviewers screened studies for eligibility. A first screening was based on titles and abstracts resulted from the search. Disagreement between the two reviewers was assessed by a third researcher, who took the final decision. All duplicates were excluded from further review process. Selected studies were screened again based on the full text by the same independent two reviewers. Again, any disagreement was judged by a third reviewer or solved by consensus.

A data extraction form was developed on an electronic spreadsheet. The form was tested on five included studies, then reassessed and refined. The following fields were included in the form and, therefore, the following data were extracted from the full text of each included study: authors, year of publication, study design (prospective or retrospective), LOE, number of shoulders treated conservatively or 
surgically, patients' age, OA classification, type of conservative treatment, surgical approach, surgical technique, functional outcomes, complications, revisions, and length of follow-up.

Data were extracted by one investigator and crosschecked by another investigator.

When data were unclear or unavailable, no attempt was made to contact authors to obtain more information regarding methodology and findings. During data extraction, references of included articles were cross-checked to search for missed studies.

\section{Quality appraisal}

The methodological assessment of included studies was performed by two authors independently. Disagreement were then solved by consensus or third part adjudication.

The Cochrane risk of bias tool [10] was used for LOE I-II studies. Each domain was judged as either, low risk of bias if all requirements were adequately fulfilled, high risk of bias if the requirements were not adequately fulfilled, and as unclear risk of bias if insufficient data for a judgment was provided.

The MINORS checklist was used to evaluate the potential risk of bias, both in comparative and in non-comparative studies, LOE III-IV [42]. The index includes 12 items, 4 of which dedicated only to comparative studies. Each item was scored 0 if not reported, 1 when reported but inadequate, and 2 when reported and adequate. The ideal score was 16 for non-comparative studies and 24 for comparative studies. Studies with a MINORS score $\leq 12$ and $\leq 20$ for noncomparative and comparative studies, respectively, were considered at high risk of bias.

\section{Statistical analysis}

Descriptive statistics was applied to summarise the data, if a pooling was not possible. Comparable outcome data from individual studies were pooled to generate summary outcomes reported as frequency-weighted values (weighted mean and standard deviation). Number of shoulders in individual studies were used to determine the weight of reported outcomes and used to calculate the weighted values.

\section{Results}

\section{Study selection}

The electronic search resulted in 996 entries. After removing the duplicates, 616 studies remained. Of these, 560 were excluded based on their abstract and 37 additional studies were excluded based on the full-text article. Nineteen studies were finally included in the review $[2,5,7,12,15,17-19$, 21, 23-26, 32-34, 37, 38, 43] (Fig. 1).

\section{Study characteristics}

Included studies reported data on 861 shoulders. Age of participants was $48.5 \pm 7.4$ years (range $19-85$ years). The mean length of follow-up was $43.8 \pm 29.9$ months (range $0.5-192$ months).

According to the LOE, only 1 study was level I [17] and 1 was level II [7], 12 studies were level III [12, 15, 18, 23-26, 32-34, 38, 43] and 5 studies were level IV [2, 5, 19, 21, 37].

Four studies [21, 25, 32, 37] reported functional results after conservative treatment, whereas 15 studies $[2,5,7,12$, $15,17-19,23,24,26,33,34,38,43]$ were focused on surgical management. No studies directly compared conservative and surgical treatments, however, seven studies [5, 7, 17-19, $24,38]$ reported a surgical approach after failure of previous conservative treatment.

Most common reported functional scores were: Visual Analogic Scale (VAS) for pain in eight studies, University of California, Los Angeles (UCLA) shoulder rating scale

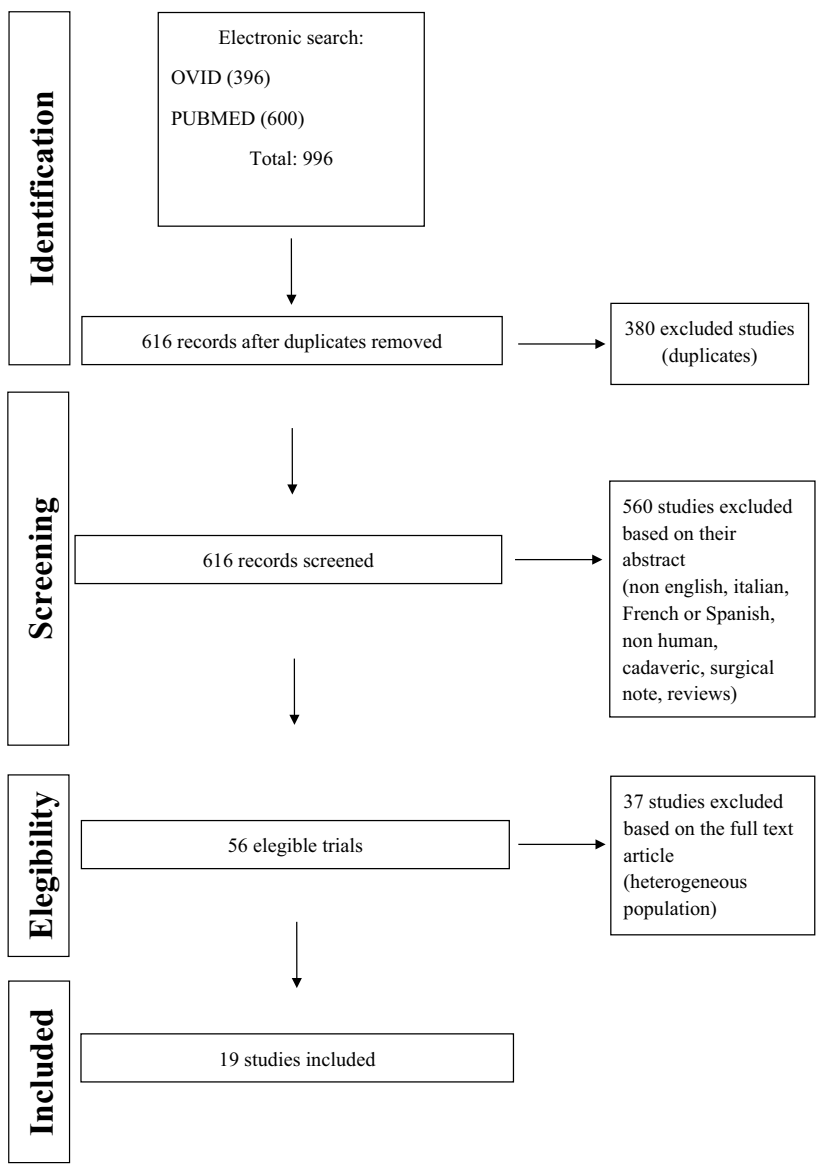

Fig. 1 Study selection based on PRISMA flow chart 
[13] in seven studies, and American Shoulder and Elbow Surgeons (ASES) society standardised shoulder assessment form [36] in seven studies, Constant score [9] in three studies, and Disabilities of the Arm, Shoulder and Hand (DASH) score [22] in two studies.

Single-study characteristics, outcomes and complications are reported in detail in Appendix 2.

\section{Conservative management}

All included studies [21, 25, 32, 37] reported results after performing intra-articular AC joint injection of corticosteroids combined with local anaesthetic. Three studies [25, $32,37]$ performed a single injection, whereas 1 study [21] deemed necessary 3 injections in 19 shoulders out of 25 to solve patients' symptoms. All studies reported functional improvement after treatment. Only Van Riet et al. [37] reported 37 drop out due to persistent pain after 1 months. All patients were offered an arthroscopic surgery. A data pooling was not possible due to the paucity of studies and heterogeneity of outcome measures.

\section{Surgical management}

Surgical treatment consisted of DCE, either arthroscopic [2, $7,12,17,18,23,24,26,33,38,43]$ or open $[12,15,17$, 19, 34, 38]. One study [5] did not report separate results for arthroscopic and open approach. However, although all studies reported functional improvement after treatment, a direct comparison between arthroscopic and open approach was not possible due to insufficient data.

\section{Risk of bias within studies}

The methodological quality of the included studies was very low.

The Cochrane risk of bias graph is reported in Fig. 2. LOE I-II studies were both judged at high risk of bias. Detailed results of the MINORS checklist are reported in Table 1. Only two non-comparative studies were not considered at high risk of bias [5, 37]; while all comparative studies $[2,12,26,32,38]$ were judged negatively.

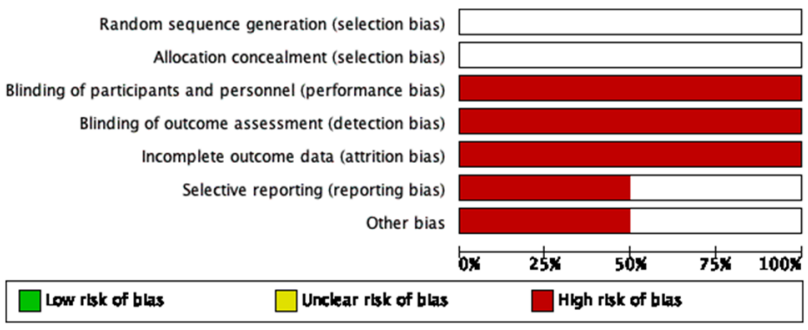

Fig. 2 Cochrane Risk of bias tool for LOE I/II studies

\section{Discussion}

The most important finding of the present study was to highlight the lack of knowledge on AC joint OA management. Although AC joint pain, due to osteoarthritic changes, is a common clinical finding, a standard treatment algorithm has not been defined yet. First-line option probably remains the conservative treatment. Included conservative studies only focused on the efficacy of steroids and local anaesthetic injections [21, 25, 32, 37], reporting high rate of success on pain relief, even in the long term [37]. However, some surgical studies also reported a previously failed combination of oral medications, physical therapy and intra-articular injections [5, 7, 17-19, 24, 38]. When a surgical approach was preferred, DCE was surely the procedure of choice. It can be performed either open or arthroscopically. Open resection was first described by Mumford in 1941 [30] and was originally used to treat chronic AC joint instability. Advance in arthroscopic techniques made nowadays the arthroscopic DCE a popular alternative, with the main goal of minimising trauma to the surrounding tissues and allowing a faster recovery. Moreover, arthroscopic resection can either be performed through an indirect (subacromial) or a direct approach [16]. Although still less common in clinical practice, the direct approach is supposed to improve the visualisation of the entire $\mathrm{AC}$ joint, to give a direct access to the joint, and to decrease bony debris in the subacromial space [26]. Only two studies [7, 26] focused on a direct comparison between direct and indirect approach. Both reported good functional results. However, while Levine et al. [26] highlighted a higher risk of superior capsule damage resulting in AC joint instability when using a direct approach, Charron et al. [7] suggested that athletes treated with the direct approach improved faster clinically and returned to sports earlier.

To summarise, it can be said that if best conservative option sounds to be a single injection of corticosteroids combined with a local anaesthetic, the situation gets confused when it comes to surgical management. Although DCE is the mainstay of treatment, surgical management can be approached as first-line option, especially when a concurrent rotator cuff tear is diagnosed or, more commonly as a second-line option after failure of conservative treatment in the setting of isolated AC joint OA. Although the DCE showed good functional outcomes and pain relief, rather than focusing on the approach, attention should be probably paid on the two critical steps of the procedure: the amount of bone resected and the preservation of the superior capsule. Even if the complication rate was very low, certainly postoperative residual AC joint instability could be an issue. Based on biomechanical results, DCE 


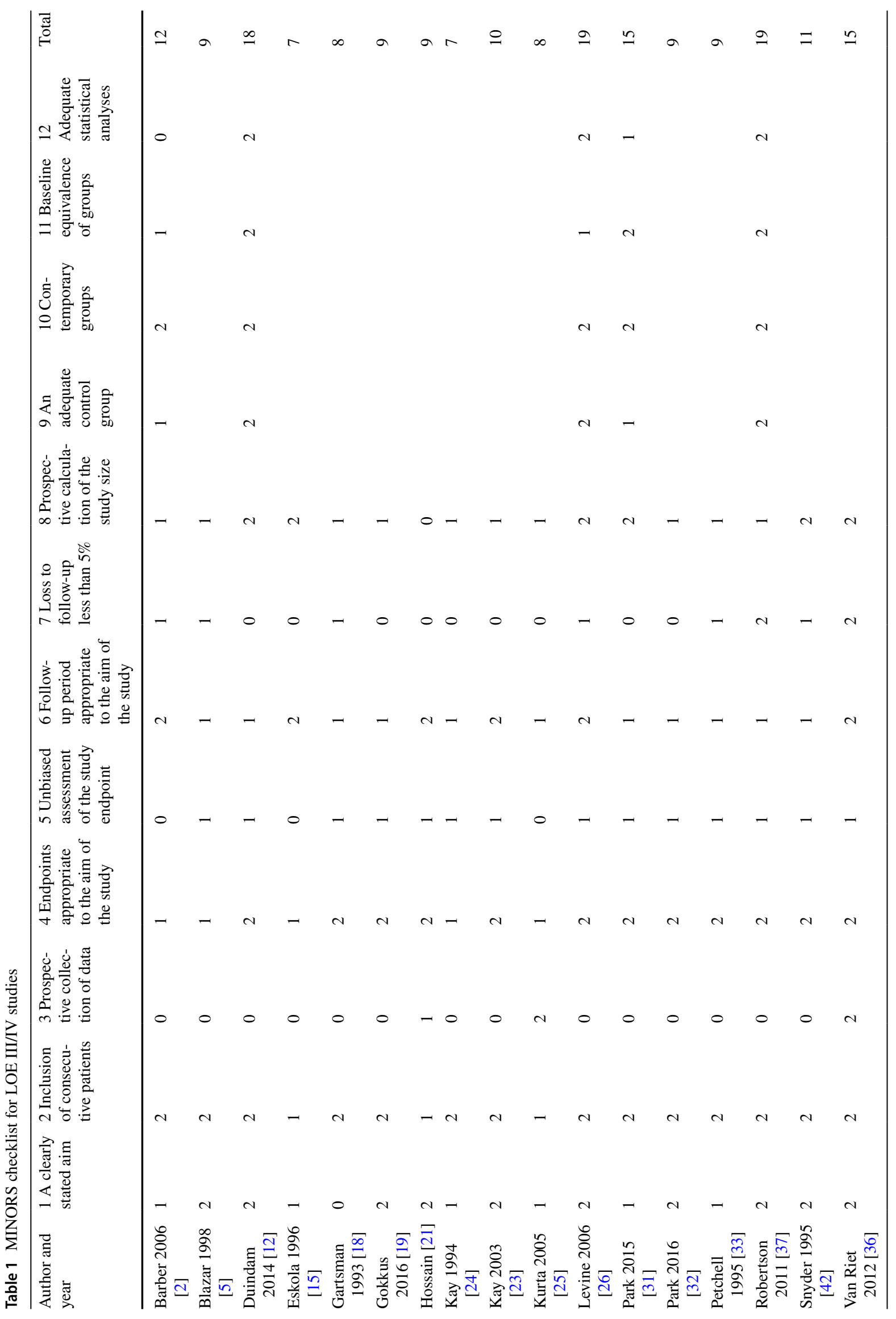


carries a risk of reduced $\mathrm{AC}$ joint stability, as resection of $10 \mathrm{~mm}$ or greater of the distal clavicle showed reduced antero-posterior stability of the AC joint [4]. Therefore, a maximum resection of $5 \mathrm{~mm}$ is recommended [4]. Moreover, Morikawa et al. recently [29] highlighted the importance of the superior capsule showing that the superior half of the AC ligament complex is the most important for both posterior and rotational stability. From a clinical standpoint, Wang et al. [46] recently conducted a metaanalysis to evaluate the effect of DCE in patients who underwent rotator cuff repair. The authors highlighted that AC joint instability was only detected in patients who underwent DCE; therefore, they clearly stated that DCE is not recommended in patients with rotator cuff tears and concomitant asymptomatic AC joint OA.

Looking at the main results of the present review, the very low methodological quality of included studies did not allow any statistical comparison neither between conservative and surgical management nor between surgical approaches. Unfortunately, most of the studies were underpowered, retrospective, and did not report sufficient data for a proper pooling. Even LOE I-II studies [7, 17] were judged at high risk of bias for improper random sequence generation as well as patient allocation and reporting bias. Although a systematic review and meta-analysis was first planned, a scoping review was than chosen as study design because available data were far away to be sufficient for a data pooling or proper statistical analysis as well as to provide definitive conclusions on the topic [31]. Systematic and scoping reviews mostly shared the same methodology; however, a scoping review is usually preferred when only a "map" of the evidence can be provided, and this seemed to be the case.

Some other recent reviews have been published. Particularly, Hohmann et al. [20] attempted a systematic review and meta-analysis focused on open versus arthroscopic AC joint resection. The authors reported similar functional and clinical outcomes, but also observed a more favourable trend towards open resection. Only four studies were included in the analysis. From a methodological standpoint, analysing together LOE I and III studies is rather questionable. Moreover, the clinical results were obtained putting in the same analysis two different functional scores (ASES and Constant), which although similar in purpose, are not the same. Finally, studies performing a combined DCE and rotator cuff repair were also included. Although previous reviews stated that DCE in the setting of rotator cuff repair showed worse results at 3-month follow-up [27], but it does not influence the outcome at 24-month follow-up [27, 46], rotator cuff repair is surely a confounding factor when focusing on the results of DCE in the setting of AC joint pain. Therefore, studies performing a combined DCE and rotator cuff repair were excluded from the present review.
Chaudhury et al. [8] recently conducted a scoping review on management of $\mathrm{AC}$ joint pain including four systematic reviews and two randomised controlled trials. A critical appraisal of included studies was attempted. The CONSORT statement [39] was used for randomised controlled trials, whereas the AMSTAR tool [41] was applied for the systematic reviews. Once again, from a methodological standpoint the choice of putting together randomised controlled trials and systematic reviews was rather singular. Moreover, the CONSORT statement is meant to be a guideline for reporting randomised controlled trials rather than evaluating their quality. Anyhow, what it sounds clear in the present study as well as in previous reviews is that evidence to support one intervention over another is rather limited because high-level studies are lacking.

Strengths of the present review compared to the previous ones mainly stay in the methodology. Strict inclusion criteria and separate quality appraisal tools for LOE I/II and LOE III/IV studies were applied, clearly resuming the current state of the art. As a matter of fact, the knowledge gaps on the topic were revealed. AC joint osteoarthritis is somehow underestimated in clinical practice; however, results of the present paper surely called more attention to the pathology. Based on the results, a single injection of corticosteroids combined with local anaesthetic could probably be considered as the first line option; however, since no direct comparisons between treatments were possible, a definitive conclusion cannot be drawn. Therefore, trials comparing the efficacy of conservative and surgical treatment strategies as well as a definitive treatment algorithm are strongly needed.

Limitations of the present review are mainly related to methodological weaknesses of included studies: heterogeneity of outcome measures and treatment protocols as well as the absence of basic information such as standard deviation in some studies made a data pooling impossible, thus preventing the possibility to establish the superiority of one intervention over another.

\section{Conclusions}

Conservative and surgical treatments are both effective in AC joint OA management. However, available data did not allow to establish the superiority of one technique over another; therefore, further high-level studies are warranted.

Funding No funding was received.

\section{Compliance with ethical standards}

Conflicts of interest The authors reports no conflicts of interest in the authorship and publication of this article. 


\section{References}

1. Armstrong A (2014) Evaluation and management of adult shoulder pain: a focus on rotator cuff disorders, acromioclavicular joint arthritis, and glenohumeral arthritis. Med Clin North Am 98:755-775

2. Barber FA (2006) Long-term results of acromioclavicular joint coplaning. Arthroscopy 22:125-129

3. Beals RK, Sauser DD (2006) Nontraumatic disorders of the clavicle. J Am Acad Orthop Surg 14:205-214

4. Beitzel K, Sablan N, Chowaniec DM, Obopilwe E, Cote MP, Arciero RA, Mazzocca AD (2012) Sequential resection of the distal clavicle and its effects on horizontal acromioclavicular joint translation. Am J Sports Med 40:681-685

5. Blazar PE, Iannotti JP, Williams GR (1998) Anteroposterior instability of the distal clavicle after distal clavicle resection. Clin Orthop Relat Res 348:114-120

6. Buss DD, Watts JD (2003) Acromioclavicular injuries in the throwing athlete. Clin Sports Med 22:327-341

7. Charron KM, Schepsis AA, Voloshin I (2007) Arthroscopic distal clavicle resection in athletes: a prospective comparison of the direct and indirect approach. Am J Sports Med 35:53-58

8. Chaudhury S, Bavan L, Rupani N, Mouyis K, Kulkarni R, Rangan A, Rees J (2018) Managing acromio-clavicular joint pain: a scoping review. Shoulder Elbow 10:4-14

9. Constant CR, Murley AH (1987) A clinical method of functional assessment of the shoulder. Clin Orthop Relat Res 214:160-164

10. Cumpston M, Li T, Page MJ, Chandler J, Welch VA, Higgins JP, Thomas J (2019) Updated guidance for trusted systematic reviews: a new edition of the Cochrane Handbook for Systematic Reviews of Interventions. Cochrane Editorial Unit (ed). Cochrane Database Syst Rev 3 10:000142

11. Docimo S, Kornitsky D, Futterman B, Elkowitz DE (2008) Surgical treatment for acromioclavicular joint osteoarthritis: patient selection, surgical options, complications, and outcome. Curr Rev Musculoskelet Med 1:154-160

12. Duindam N, Kuiper JWP, Hoozemans MJM, Burger BJ (2014) Comparison between open and arthroscopic procedures for lateral clavicle resection. Int Orthop 38:783-789

13. Ellman H, Hanker G, Bayer M (1986) Repair of the rotator cuff. End-result study of factors influencing reconstruction. J Bone Joint Surg Am 68:1136-1144

14. Ernberg LA, Potter HG (2003) Radiographic evaluation of the acromioclavicular and sternoclavicular joints. Clin Sports Med 22:255-275

15. Eskola A, Santavirta S, Viljakka HT, Wirta J, Partio TE, Hoikka V (1996) The results of operative resection of the lateral end of the clavicle. J Bone Joint Surg Am 78:584-587

16. Flatow EL, Duralde XA, Nicholson GP, Pollock RG, Bigliani LU (1995) Arthroscopic resection of the distal clavicle with a superior approach. J Shoulder Elbow Surg 4:41-50

17. Freedman BA, Javernick MA, O'Brien FP, Ross AE, Doukas WC (2007) Arthroscopic versus open distal clavicle excision: comparative results at six months and one year from a randomized, prospective clinical trial. J Shoulder Elbow Surg 16:413-418

18. Gartsman GM (1993) Arthroscopic resection of the acromioclavicular joint. Am J Sports Med 21:71-77

19. Gokkus K, Saylik M, Atmaca H, Sagtas E, Aydin AT (2016) Limited distal clavicle excision of acromioclavicular joint osteoarthritis. Orthop Traumatol Surg Res 102:311-318

20. Hohmann E, Tetsworth K, Glatt V (2019) Open versus arthroscopic acromioclavicular joint resection: a systematic review and meta-analysis. Arch Orthop Trauma Surg 139:685-694

21. Hossain S, Jacobs LGH, Hashmi R (2008) The long-term effectiveness of steroid injections in primary acromioclavicular joint arthritis: a five-year prospective study. J Shoulder Elbow Surg 17:535-538

22. Hudak PL, Amadio PC, Bombardier C (1996) Development of an upper extremity outcome measure: the DASH (disabilities of the arm, shoulder and hand). The Upper Extremity Collaborative Group (UECG). Am J Ind Med 29:602-608

23. Kay SP, Dragoo JL, Lee R (2003) Long-term results of arthroscopic resection of the distal clavicle with concomitant subacromial decompression. Arthroscopy 19:805-809

24. Kay SP, Ellman H, Harris E (1994) Arthroscopic distal clavicle excision. Technique and early results. Clin Orthop Relat Res 301:181-184

25. Kurta I, Datir S, Dove M, Rahmatalla A, Wynn-Jones C, Maffulli N (2005) The short term effects of a single corticosteroid injection on the range of motion of the shoulder in patients with isolated acromioclavicular joint arthropathy. Acta Orthop Belg 71:656-661

26. Levine WN, Soong M, Ahmad CS, Blaine TA, Bigliani LU (2006) Arthroscopic distal clavicle resection: a comparison of bursal and direct approaches. Arthroscopy 22:516-520

27. Livingstone A, Asaid R, Moaveni AK (2019) Is routine distal clavicle resection necessary in rotator cuff repair surgery? A systematic review and meta-analysis. Shoulder Elbow 11:39-45

28. Mall NA, Foley E, Chalmers PN, Cole BJ, Romeo AA, Bach BR (2013) Degenerative joint disease of the acromioclavicular joint: a review. Am J Sports Med 41:2684-2692

29. Morikawa D, Dyrna F, Cote MP, Johnson JD, Obopilwe E, Imhoff FB, Beitzel K, Mazzocca AD, Scheiderer B (2019) Repair of the entire superior acromioclavicular ligament complex best restores posterior translation and rotational stability. Knee Surg Sports Traumatol Arthrosc 27:3764-3770

30. Mumford EB (1941) Acromioclavicular dislocation: a new operative treatment. J Bone Joint Surg 4:799-802

31. Munn Z, Peters MDJ, Stern C, Tufanaru C, McArthur A, Aromataris E (2018) Systematic review or scoping review? Guidance for authors when choosing between a systematic or scoping review approach. BMC Med Res Methodol 18:143

32. Park KD, Kim TK, Lee J, Lee WY, Ahn JK, Park Y (2015) Palpation versus ultrasound-guided acromioclavicular joint intra-articular corticosteroid injections: a retrospective comparative clinical study. Pain Phys 18:333-341

33. Park T-S, Lee K-W (2016) Arthroscopic resection of the distal clavicle in osteoarthritis of the acromioclavicular joint. Indian J Orthop 50:379-383

34. Petchell JF, Sonnabend DH, Hughes JS (1995) Distal clavicular excision: a detailed functional assessment. Aust N Z J Surg 65:262-266

35. Precerutti M, Formica M, Bonardi M, Peroni C, Calciati F (2020) Acromioclavicular osteoarthritis and shoulder pain: a review of the role of ultrasonography. J Ultrasound 23:317-325

36. Richards RR, An KN, Bigliani LU, Friedman RJ, Gartsman GM, Gristina AG, Iannotti JP, Mow VC, Sidles JA, Zuckerman JD (1994) A standardized method for the assessment of shoulder function. J Shoulder Elbow Surg 3:347-352

37. van Riet RP, Goehre T, Bell SN (2012) The long term effect of an intra-articular injection of corticosteroids in the acromioclavicular joint. J Shoulder Elbow Surg 21:376-379

38. Robertson WJ, Griffith MH, Carroll K, O'Donnell T, Gill TJ (2011) Arthroscopic versus open distal clavicle excision: a comparative assessment at intermediate-term follow-up. Am J Sports Med 39:2415-2420

39. Schulz KF, Altman DG, Moher D (2010) CONSORT 2010 Statement: updated guidelines for reporting parallel group randomised trials. J Pharmacol Pharmacother 1:100-107

40. Shaffer BS (1999) Painful conditions of the acromioclavicular joint. J Am Acad Orthop Surg 7:176-188 
41. Shea BJ, Grimshaw JM, Wells GA, Boers M, Andersson N, Hamel C, Porter AC, Tugwell P, Moher D, Bouter LM (2007) Development of AMSTAR: a measurement tool to assess the methodological quality of systematic reviews. BMC Med Res Methodol 7:10

42. Slim K, Nini E, Forestier D, Kwiatkowski F, Panis Y, Chipponi J (2003) Methodological index for non-randomized studies (minors): development and validation of a new instrument. ANZ J Surg 73:712-716

43. Snyder SJ, Banas MP, Karzel RP (1995) The arthroscopic Mumford procedure: an analysis of results. Arthroscopy 11:157-164

44. Stein BE, Wiater JM, Pfaff HC, Bigliani LU, Levine WN (2001) Detection of acromioclavicular joint pathology in asymptomatic shoulders with magnetic resonance imaging. J Shoulder Elbow Surg 10:204-208

45. Tricco AC, Lillie E, Zarin W, O'Brien KK, Colquhoun H, Levac D, Moher D, Peters MDJ, Horsley T, Weeks L, Hempel S, Akl EA,
Chang C, McGowan J, Stewart L, Hartling L, Aldcroft A, Wilson MG, Garritty C, Lewin S, Godfrey CM, Macdonald MT, Langlois EV, Soares-Weiser K, Moriarty J, Clifford T, Tunçalp Ö, Straus SE (2018) PRISMA extension for scoping reviews (PRISMAScR): checklist and explanation. Ann Intern Med 169:467

46. Wang J, Ma J-X, Zhu S-W, Jia H-B, Ma X-L (2018) Does distal clavicle resection decrease pain or improve shoulder function in patients with acromioclavicular joint arthritis and rotator cuff tears? A meta-analysis. Clin Orthop Relat Res 476:2402-2414

Publisher's Note Springer Nature remains neutral with regard to jurisdictional claims in published maps and institutional affiliations. 